\title{
Single Domain Wall Propagation at Low Fields
}

\author{
J. KOSTYK ${ }^{a, *}$, R. VARGA ${ }^{a}$, M. VAZQUEZ $^{b}$ \\ ${ }^{a}$ Institute of Physics, Faculty of Sciences, P. J. Safarik University, Park Angelinum 9, 04154 Košice, Slovakia \\ ${ }^{b}$ Instituto de Ciencia de Materiales, CSIC 28049 Madrid, Spain
}

\begin{abstract}
We have studied the domain wall propagation at low fields regime in thin glass-coated microwire of composition $\mathrm{Fe}_{77.5} \mathrm{Si}_{7.5} \mathrm{~B}_{15}$. It is shown, that power law describes domain wall dynamics at low fields. Such behaviour results from the interaction of the propagating domain wall with the defects present in the material. At high fields, the domain wall mobility becomes negative. This can be explained as a result of domain structure relaxation. The exponent $q$ from power law, which determines the domain wall shape, has a value of 0.19 for both cases, without applied stress and with applied stress of $20 \mathrm{MPa}$. This means, that domain wall shape is flexible in both measurements.
\end{abstract}

DOI: $10.12693 /$ APhysPolA.126.30

PACS: 75.60.Ch, 75.60.Ej, 75.78.Fg

\section{Introduction}

Domain wall propagation is used in many magnetic devices such as magnetic random access memory, spintronics and sensor devices [1]. The speed of such devices depends on the velocity of the magnetic domain wall. One of the problems is understanding the domain wall propagation through a real material containing defects. This will help us in controlling of such devices.

Amorphous glass-coated microwires with positive magnetostriction prepared by Taylor-Ulitovski method are characterized by depinning and subsequent propagation of the single closure domain wall in one large Barkhausen jump. Because of amorphous nature, they contain defects (local density fluctuations), which act as pinning centers. That is why the magnetic microwires are ideal to study the single domain wall dynamics in defective material.

Dynamics of domain wall in material, which contains defects, is similar to the body oscillation under external force in viscous medium [2]. If we assume constant velocity, we can express dependence between domain wall velocity and applied magnetic field by linear equation:

$$
v=S\left(H-H_{t}\right),
$$

where $S$ is the mobility of domain wall and $H_{t}$ is the critical propagation field. Specially for amorphous glasscoated microwires, critical propagation field became very interesting parameter, after making first measurements of domain wall dynamics, because of its negative value. These results reflect theoretical possibility of domain wall propagation at zero field. Therefore, the new aim was to measure domain wall propagation at low fields.

Later, measurements of domain wall dynamics at low fields were performed and it was shown, that the classical linear dependence of the domain wall velocity on the applied magnetic field in microwires is not valid at low fields, where domain wall dynamics can be successfully

\footnotetext{
*corresponding author; e-mail: kostyk@centrum.sk
}

described by a power law:

$$
v=S^{\prime}\left(H-H_{0}\right)^{q},
$$

where $S^{\prime}$ is the domain wall mobility parameter, $H_{0}$ is the dynamic coercive field and $q$ is the power exponent. Such behaviour results from the interaction of the propagating domain wall with the defects present in the material. It was also shown, from measurements of temperature dependence of $q$, that the value of power exponent is determined by the domain wall shape. The mobility parameter $S^{\prime}$ is proportional to the mobility $S$ from equation (1) and hence it is field independent [3, 4].

\section{Experimental}

We have studied domain wall dynamics on the amorphous glass-coated microwire of composition $\mathrm{Fe}_{77.5} \mathrm{Si}_{7.5} \mathrm{~B}_{15}$. The microwire was $10.5 \mathrm{~cm}$ long, with $11 \mu \mathrm{m}$ metallic nucleus diameter and total diameter of $29 \mu \mathrm{m}$. It was prepared by Taylor-Ulitovsky method. Sixtus-Tonks like experiment was used for domain wall dynamics measurements. More details can be found elsewhere $[3,4]$.

The measurements have been performed at $77 \mathrm{~K}$ under the applied tensile mechanical stress of up to $20 \mathrm{MPa}$. The frequency of applied magnetic field was $10 \mathrm{~Hz}$.

\section{Results and discussion}

The domain wall dynamics measured at $77 \mathrm{~K}$ looks very interesting (Fig. 1). Firstly, the domain wall velocity increases steeply at low fields $(<400 \mathrm{~A} / \mathrm{m})$, then (above $500 \mathrm{~A} / \mathrm{m}$ ) it decreases linearly with applied magnetic field.

The negative domain wall mobility is a result of the stabilization of the domain structure. It appears at low frequency (where there is enough time for relaxation between two subsequent domain wall propagations) [5]. Such a regime can be controlled by the frequency of applied field and measuring temperature [5].

However, we have focused on the low field regime. It has been shown, that at low fields that domain wall dynamics deviates from the linear dependence of the velocity on applied field given by Eq. $1[4,8,9]$. Instead the 
domain wall dynamics at low fields is described by power law (Eq. 2).

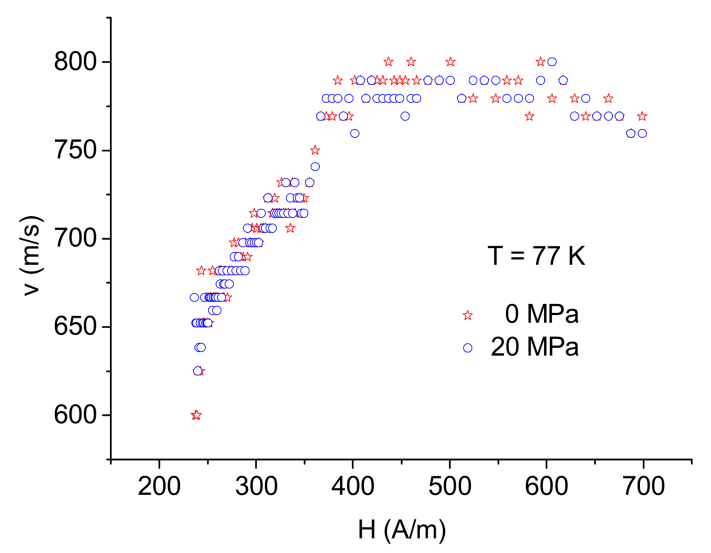

Fig. 1. Velocity of domain wall as a function of applied magnetic field at $77 \mathrm{~K}$ and different applied stresses.

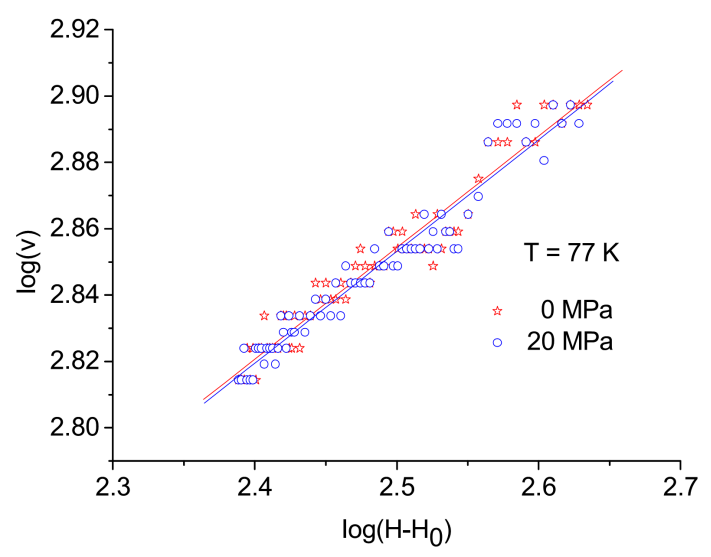

Fig. 2. Velocity of domain wall as a function of applied magnetic field at $77 \mathrm{~K}$ and different applied stresses in logarithmic scale.

Using log-log representation of $v \sim f(H)$ (Fig. 2) we can obtain values of parameters $S^{\prime}$ and $q$, (see Tab. 1 ).

The domain wall potential consists of two contributions: first one is the long-range magnetoelastic contribution; second one is the short-range contribution, which depends on domain wall interaction with defects in the material. Domain wall dynamics in local scale (one defect) can be described as:

$$
v=S\left(H-\left(H_{l r}+H_{p}\right)\right),
$$

where $H_{l r}$ is the field that arises from the long range interaction (magnetoelastic in our case) of the domain wall with the stresses and $H_{p}$ is the pinning field of defect. Although equation (3) is linear, it describes only local situation. The value of pinning field fluctuates through the microwire and this results in an average velocity that is given by a power law (Eq. 2) [6, 7]. It is known that power exponent $q$ determines the domain wall shape [4]. At high temperatures, the magnetoelastic contribution is dominant and the domain wall pinning is small. There- fore, domain wall has rigid planar shape for minimizing its energy, giving the value of power exponent of $q \sim 0.5$. At low temperatures, the defects lose their mobility, the pinning becomes strong and for the domain wall it is better to become flexible. In such case the value of $q$ decreases $[3,4]$.

In our experiment $q$ is about 0.19 for both measurements (without the stress and with the applied stress of $20 \mathrm{MPa}$ ). This points to the flexible domain wall shape that does not vary with the stress application. Consequently, we can assume, that power exponent does not depend on magnetoelastic contribution and is only a function of domain wall pinning on local defects.

Power law parameters fitted to Eq. 2

TABLE I

\begin{tabular}{c|c|c|c}
\hline \hline$\sigma(\mathrm{MPa})$ & $S$ & $H_{0}$ & $q$ \\
\hline 0 & $272 \pm 8.69$ & $145 \pm 5.19$ & $0.189 \pm 0.006$ \\
\hline 20 & $273 \pm 6.76$ & $146 \pm 4.34$ & $0.186 \pm 0.004$
\end{tabular}

\section{Conclusions}

The domain wall dynamics was studied in thin glasscoated microwire at low fields, where the power law describes the domain wall propagation. Such behaviour results from the interaction of the propagating domain wall with the defects present in the material. The power exponent $q$ is determined by the domain wall shape. Value of $q$ is approximately 0.19 in both our measurements, without the stress and with the stress of $20 \mathrm{MPa}$ applied. This means, that domain wall shape is flexible in both cases and it was not changed by the application of stress.

\section{Acknowledgments}

This work was supported by the project NanoCEXmat Nr. ITMS 26220120019, Slovak VEGA grant. No. 1/0060/13, APVV-0027-11 and APVV-0266-10.

\section{References}

[1] D.A. Allwood, G. Xiong, C.C. Faulkner, D. Atiknson, D. Petit, R.P. Cowburn, Science 309, 1688 (2005).

[2] C.W. Chen, Magnetism and metallurgy of soft magnetic materials Dover Pub., Inc., New York 1986.

[3] R. Varga, Y. Kostyk, A. Zhukov, M. Vazquez, J. of Non-Crystal. Solids 354, 5101 (2008).

[4] R. Varga, J. Torrejon, Y. Kostyk, K.L. Garcia, G. Infantes, G. Badini, M. Vazquez, J.Phys.: Cond. Matter 20, 445215 (2008).

[5] R. Varga, G. Infante, K. Richter, M. Vazquez, Phys. Stat. Sol. A 208, 509 (2011).

[6] G. Durin, The Barkhaussen effect in: Science of Hysteresis Vol. II, G. Bertotti, I. Mayergoyz, Acad. Press, Oxford 2006.

[7] B. Alessandro, C. Beatrice, G. Bertotti, A. Montorsi, J. Appl. Phys. 68, 2901 (1990).

[8] J. Ziman, V. Šuhajová, M. Kladivová, Physica B 407, 3905 (2012).

[9] J. Gonzalez, A. Chizik, A. Zhukov, J.M. Blanco, Phys. Stat. Sol. A 208, 502 (2011). 